Running Head: Consciousness and brain-heart interactions

\title{
Brain-heart interactions reveal consciousness in
}

\section{non-communicating patients}

Federico Raimondo ${ }^{1,2,3,4} \mathbb{D}^{3}$; Benjamin Rohaut MD, $\mathrm{PhD}^{5,6}$ (D) ; Athena Demertzi PhD ${ }^{3,5}$; Melanie Valente ${ }^{3,5}$; Denis Engemann ${ }^{3,5,7,8}$; Moti Salti PhD ${ }^{9}$; Diego Fernandez Slezak PhD ${ }^{1,2}$; Lionel Naccache MD, $\mathrm{PhD}^{3,4,5,6,10}$; Jacobo D. Sitt MD, $\mathrm{PhD}^{3,5}$

1. Universidad de Buenos Aires, Facultad de Ciencias Exactas y Naturales, Departamento de Computación, C1428EGA, Ciudad Autónoma de Buenos Aires, Argentina.

2. CONICET - Universidad de Buenos Aires, Instituto de Investigación en Ciencias de la Computación, Godoy Cruz 2290, C1425FQB, Ciudad Autónoma de Buenos Aires, Argentina

3. Institut du Cerveau et de la Moelle épinière, 47 bd de l'Hôpital, 75013, Paris, France

4. Sorbonne Universités, UPMC Université Paris 06, Faculté de Médecine Pitié-Salpêtrière, 91-105 bd de l'Hôpital, 75013, Paris, France

5. INSERM, U 1127, 47 bd de l'Hôpital, 75013, Paris, France

6. Department of Neurology, Groupe hospitalier Pitié-Salpêtrière, AP-HP, 47-83 bd de l'Hôpital, 75013, Paris, France

7. Parietal project-team, INRIA Saclay - ile de France, France

8. Cognitive Neuroimaging Unit, CEA DSV/I2BM, INSERM, Université Paris-Sud, Université Paris-Saclay, NeuroSpin center, 91191 Gif sur Yvette, France

9. Zlotowski Center for Neuroscience and Brain Imaging Research Center, Ben-Gurion University of the Negev, PO Box 653, Beer-Sheva, 84105, Israel

10. Department of Neurophysiology, Groupe hospitalier Pitié-Salpêtrière, AP-HP, 47-83 bd de I'Hôpital, 75013, Paris, France

\section{Corresponding author}

Jacobo Diego Sitt, MD, PhD. email: jacobo.sitt@inserm.fr

Institut du Cerveau et de la Moelle épinière - ICM

Hôpital Pitié Salpêtrière, Paris, France

Tel: +33 (0) 157274317

Running head character count: 42

Title Character Count: 75

Word count in abstract: 249

Word count in introduction: 444

Word count in discussion: 1341

Word count in full body: 5076

Number of figures: 6 (incuding color figures)

Number of color figures: 2

Number of tables: 1

This article has been accepted for publication and undergone full peer review but has not been through the copyediting, typesetting, pagination and proofreading process which may lead to differences between this version and the Version of Record. Please cite this article as an 'Accepted Article', doi: 10.1002/ana.25045 


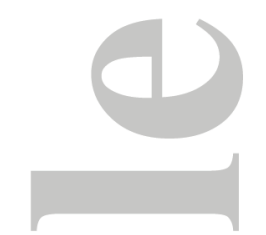

Abstract

Objective

We here aimed at characterizing heart-brain interactions in patients with disorders of consciousness. We tested how this information impacts data-driven classification between unresponsive and minimally conscious patients.

Methods

A cohort of 127 patients in vegetative state/unresponsive wakefulness syndrome (VS/UWS, $n=70)$ and minimally conscious state (MCS, $n=57)$ were presented with the 'Local-Global' auditory oddball paradigm, which distinguishes two levels of processing: short-term deviation of local auditory regularities and global long-term rule violations. In addition to previously validated markers of consciousness extracted from electroencephalograms (EEG), we computed autonomic cardiac markers, such as heart rate and variability (HR, HRV), and cardiac cycle phase-shifts triggered by the processing of the auditory stimuli.

\section{Results}

HR and HRV were similar in patients across groups. The cardiac cycle was not sensitive to the processing of local regularities in either the VS/UWS or MCS patients. In contrast, global regularities induced a phase-shift of the cardiac cycle exclusively in the MCS group. The interval between the auditory stimulation and the following R-peak was significantly shortened in MCS when the auditory rule was violated. When the information of the cardiac cycle modulations and other consciousness-related EEG markers were combined, singlepatient classification performance was enhanced compared to classification with solely EEG markers.

\section{Interpretation}

Our work shows a link between residual cognitive processing and the modulation of autonomic somatic markers. These results open a new window to evaluate patients with disorders of consciousness via the embodied paradigm, according to which body-brain functions contribute to a holistic approach to conscious processing. 


\section{Introduction}

Patients with disorders of consciousness (DOC) are characterized by preserved wakefulness in the absence of clear evidence of awareness such that they remain unable to communicate with their surroundings ${ }^{1}$. For example, patients in a vegetative state/unresponsive wakefulness syndrome (VS/UWS) open their eyes but they do not show conscious responses to sensory stimulation ${ }^{2}$. When patients exhibit signs of fluctuating yet reproducible remnants of non-reflex behavior, such as visual pursuit, they are considered to be in a minimally conscious state (MCS) ${ }^{3}$. The diagnostic assessment of patients with DOC is mainly based on the observation of motor and oculomotor behaviors at the bedside ${ }^{4}$. The evaluation of non-reflex behavior, however, is not straightforward as patients can fluctuate in terms of vigilance, may suffer from cognitive and/or sensory impairments, from small or easily exhausted motor activity and pain, which may lead in the underestimation of the level of consciousness ${ }^{5,6}$. Previous work employing data-driven analyses with neuroimaging and neurophysiological tools suggest relatively accurate patient diagnosis ${ }^{7-10}$ and prediction of clinical outcome ${ }^{11}$.

Aside this neuro-centric approach, classic ${ }^{12,13}$ and more recent studies in interoception ${ }^{14-18}$ in healthy subjects demonstrate that brain-modulation of peripheral body functions can be affected by concomitant cognitive processes. Therefore, such 'brain-body' interaction might be relevant to evaluate consciousness states in DOC patients. Cardiac activity is a peripheral body signal that has been linked to cognitive processes. For example, 'bradycardia of attention' refers to the effect of heartbeat frequency deceleration when the subject is engaged in an active cognitive task (such as target detection or auditory odd-ball counting $)^{19}$. As regards patients with DOC, previous work has shown that cardiac autonomic markers, such as Heart Rate (HR) and Heart Rate Variability (HRV), were markers of autonomic system malfunction (dysautonomia) after traumatic brain injury ${ }^{20,21}$. However, the link between these autonomic markers and conscious cognitive processing in DOC patients remains unknown.

Here, we aimed at (1) characterizing consciousness state of DOC patients by means of heart-brain interactions and (2) determining if the electrocardiogram (EKG)-extracted information may complement the single-patient EEG diagnosis of consciousness state. For the first objective, we quantified the modulation of cardiac cycle during an auditory 
stimulation protocol, known as the 'Local-Global' paradigm, which is designed to test two hierarchical levels of processing of auditory regularities ${ }^{22}$. For the second objective, we contrasted the performance of multivariate patient classification of the state-ofconsciousness at the single-patient level using solely EEG markers, and combining EEG and cardiac cycle modulation markers. We hypothesized a cardiac cycle modulation in the group of MCS patients and that this modulation will carry partially independent information about the state of consciousness, reflected in the form of an enhanced classification performance.

\section{Methods}

\section{Subject and Patients}

Patients admitted for consciousness evaluation at the Neurological Department of the Pitié-Salpêtrière hospital, Paris between February 2008 and April 2015 were included. Informed consent was signed by the patients' legal representatives. The protocol conformed to French regulation and the Declaration of Helsinki and was approved by the ethics committee CPP Ile de France 1 (Paris, France). The neurological evaluation of the patients' disorders of consciousness was performed by trained clinicians, including the Coma Recovery Scale-Revised (CRS-R). The CRS-R scoring varies from 0-23 and is based on the presence or absence of response on a set of hierarchically ordered items testing auditory, visual, motor, oro-motor, communication and arousal functions ${ }^{4}$. Behavioral evaluations were performed systematically before each EEG recording.

In the present study, we aimed at characterizing the cardiac cycle in relation to the state of consciousness as a post-hoc analysis. No EKG was available during the EEG evaluations. As a consequence, EKG timeseries were obtained using independent component analysis (ICA) on the EEG recordings for each patient. The current analysis only used the temporal location of the $\mathrm{R}$ wave peaks.

From the 259 patients originally assessed with EEG (130 VS/UWS, 129 MCS), 132 patients (51\%; $60 \mathrm{VS} / \mathrm{UWS}, 72 \mathrm{MCS}$ ) were rejected due to the lack of a clear EEG recording or EKG reconstructed source that produced at least 40 samples for each stimulation block type. There was no differences between the included and excluded patients in terms of diagnostic state $\left(\chi^{2}(1, N=259)=2.07, p=0.15\right)$ and $\operatorname{sex}\left(\chi^{2}(1, N=259)=0.21, p=0.64\right)$. Included patients were older than excluded patients ( $48 \pm 18$ vs $44 \pm 17$ years; $W=6701, p=0.04$ ) and more patients 
were of anoxic as compared to traumatic injuries in the included group compared to the excluded group $\left(\chi^{2}(4, N=259)=12.84, p=0.01\right)$.

A final cohort of 127 (49\%) patients remained: 70 in VS/UWS (20 females, mean age $45 \pm 19$, range 17-80, 12 traumatic, 21 assessed in a chronic setting, i.e., > 1 month postinsult), and 57 in MCS (17 females, mean age 52 \pm 16 , range 21-79, 13 traumatic, 17 assessed in a chronic setting). Patient groups did not differ in terms of gender $\left(\chi^{2}(1, N=127)=6.2 e^{-31}\right.$, $p=1)$, etiologies $\left(\chi^{2}(4, N=127)=9.4, p=0.051\right)$ and chronicity $\left(\chi^{2}(1, N=127)=2 e^{-30}, p=1\right)$. MCS patients were older than VS/UWS ( $52 \pm 16$ vs 45 $\pm 19 ; \mathrm{W}=2435, \mathrm{p}=0.03)$. No patient had any history of cervical spinal cord injury or symptoms of autonomic dysfunction (e.g. hemodynamic instability, HR abnormal variability) at the time of EEG recording.

\section{Auditory Stimulation and EEG}

Cognitive processing was prompted by means of the EEG-based auditory Local-Global paradigm ${ }^{22}$. The Local-Global paradigm is characterized by two embedded levels of auditory regularities (Figure 1A). Each trial is formed by 5 consecutive sounds lasting $50 \mathrm{~ms}$, with a 150 ms gap between the sounds' onsets and an inter-trial interval ranging from 1350 to $1650 \mathrm{~ms}$. The fifth sound can be either equal or different to the first four, this defines whether the trial is standard or deviant at a local (or within-trial) level. The second level of regularities is defined across trials (or at a global level), frequent trials (80\%) define the regularity, and rare ones (20\%) violate this regularity. Two types of stimulation blocks are played to the subjects: in the XX blocks, the frequent stimulus corresponds to five equal sounds (LSGS: Local Standard and Global Standard). In contrast, the infrequent stimulus corresponds to four equal sounds followed by a fifth different sound (LDGD: Local Deviant and Global Deviant). In the $X Y$ blocks, the frequent stimulus corresponds to four equal sounds and a fifth different sound (LDGS: Local Deviant and Global Standard). The infrequent stimulus corresponds to five equal sounds (LSGD: Local Standard and Global Deviant) (Figure 1A). The Local effect is quantified by contrasting all local deviant trials ( $L D: L D G S+L D G D)$ versus all local standard trials ( $L S: \angle S G S+L S G D$ ). The Global effect is quantified by contrasting all global deviant trials (GD: $L S G D+L D G D$ ) versus all global standard trials (GS: $L S G S+L D G S$ ). Patients were stimulated vocally between each stimulation block ( $/ 3.5 \mathrm{~min}$, task instruction) and with tactile stimulations if patients appeared asleep (pressure as recommended in the "arousal facilitation protocol" in the CRS-R). A mask was applied on the eyes to normalize 
the eyes open/closed across patients. EEG recordings were performed using a NetAmps 300 amplifier (EGI) at a sampling rate of $250 \mathrm{~Hz}$ with a 256 electrodes HydroCel Geodesic Sensor Net (HCGSN) referenced to the vertex.

\section{EKG extraction from EEG}

In the absence of direct recordings of cardiac activity, EKG was extracted from the EEG using ICA. The independent components (IC) corresponding to the EKG were selected by visual inspection based on the spatial and temporal representation of the QRS complex. Raw EEG data was first filtered using an $8^{\text {th }}$ order low-pass butterworth filter at $45 \mathrm{~Hz}$ and a $4^{\text {th }}$ order high-pass filter at $0.5 \mathrm{~Hz}$ (Figure 2A). Secondly, we computed three different ICA decompositions: (1) FastICA ${ }^{23}$ parametrized to obtain the components that explain $99 \%$ of the variance and computed from raw filtered data, (2) INFOMAX ${ }^{24,25}$ parametrized to obtain 256 components from raw filtered data and (3) INFOMAX in combination to artifact channels rejection. Individual channels were removed when the temporal variance was more than 3 standard deviations away from the mean of the variance of the rest of the channels. The IC with the EKG information was selected based on the timeseries and the weights' topographies by visual inspection (Figure $2 \mathrm{~B}$ ). The selected timeseries had to clearly contain the R-peak corresponding to the QRS complex. The R-peak had to be easily detected by using a simple threshold. The corresponding topography had to concentrate the mixing weights on the frontal right and posterior left electrodes. These electrodes are located in the right cheek, left maxillary junction and underneath the left mastoid, as depicted by previous studies on cardiac electrical fields ${ }^{26}$. We then picked the algorithm that presented the clearest decomposition, usually the one with the highest rank in descending order of explained variance. Finally, R-peaks onsets were obtained automatically by the algorithm described in Elgendi et $\mathrm{al}^{27}$. Subjects for which the EKG component was unclear were excluded from the analysis. Exclusion criteria was set to any of: EKG reconstructed signal with no clear R-peaks, detection failure by the automatic algorithm, or a topography of corresponding weights with a mix of peripheral and central electrodes.

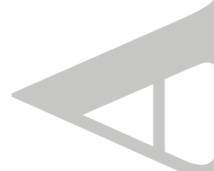

\section{Baseline cardiac activity}

The overall heart rate (HR) was computed by averaging the differences between consecutive R-peaks (RR Intervals, Figure 1B) during the whole recording. Following the 
method described in Deboer et al. ${ }^{28}$, heart rate variability (HRV) spectral variables were obtained by computing the power spectrum decomposition on the point events timeseries from the detected R-peaks. Power Spectral Density was estimated in whole recording using Welch's method with 32768 samples (131.072s) per segment and 28672 samples (114.688s) overlap using a Hanning window. HRV variables were extracted from the sum of the spectral power in three frequency bands: 1) very low frequencies (VLF, range $0-0.04 \mathrm{~Hz}), 2$ ) low frequencies (LF, range $0.04-0.15 \mathrm{~Hz}$ ) and 3) high frequencies $(\mathrm{HF}$, range $0.15-0.4 \mathrm{~Hz})$.

\section{R-peak locked EEG evoked responses}

EEG recordings were filtered as previously described, segmented from -200ms to $600 \mathrm{~ms}$ relative to the onset of the R-peak and baseline corrected using the $200 \mathrm{~ms}$ long window before R-peak. Bad channels and trials were rejected based on peak-to-peak amplitude exceeding $100 \mu \mathrm{V}$. Bad channels were interpolated. The remaining trials were averaged. We performed a group analysis and obtained the mean evoked response for each group, and contrasted the VS/UWS mean evoked activity to the MCS one. Statistics on EEG responses were done using non-parametric cluster corrected permutation test ${ }^{29}$.

\section{Markers of cardiac cycle modulation induced by the auditory stimulation protocol}

To evaluate potential phase shifts in the cardiac cycle associated to the processing of different types of auditory stimuli, two intervals temporally locked to the onset of the fifth sound were defined (Figure 1B): (1) the PRE interval: the interval between the heartbeat (defined by the location of the R-peak) preceding the stimulus and the onset of the auditory stimulation, and (2) the POST interval: the interval between the stimulus onset and the following heartbeat. All time intervals were then labelled according to the contained auditory stimulation following the Local-Global paradigm (XX block: LSGS or LDGD; XY block: LDGS or LSGD). Finally, In order to avoid using peaks without a clearly defined temporal association to a given heartbeat (and not the previous or following one), we restricted the analysis to the trials in which both the PRE and POST intervals were between 20 and 600 milliseconds. A mean of $520 \pm 150$ trials per subject were included while $135 \pm 100$ trials were rejected (20 $\pm 13 \%)$. A repeated measures Bayesian ANOVA was computed for each interval using the ratio of rejected trials as the study variable and the trial label and clinical state as factors. All the models including the clinical state factor presented evidence for no 
difference (PRE BF $01>=2.35 ;$ POST $B F_{01}>=2.61$ ). When the models included the trial type factor, the test showed strong evidence for no difference $\left(P R E B F_{01}>=39.19 ; P O S T\right.$ $\left.\mathrm{BF}_{01}>=45.17\right)$.

To test if conscious processing of auditory regularities affects the ongoing cardiac activity, we analyzed the PRE and POST stimulus intervals for each group of subjects in relation with the type of trials. For the Local effect, each subject mean of the PRE and POST intervals corresponding to LD trials were subtracted from the mean of the LS ones. Similarly, for the Global effect, the mean of the PRE and POST intervals corresponding to GD trials were subtracted from the mean of GS ones.

\section{Multivariate Pattern Analysis (MVPA)}

In order to analyze the relevance and independence of the markers to the diagnosis of DOC, we used Multivariate Pattern Analysis in combination with wrappers algorithms for feature selection ${ }^{30}$. This method consists on training classifiers with different set of features and comparing the obtained performance. Based on the performance comparisons, a set of features can be defined as (1) strongly or weakly relevant when they are partially independent and contribute to an optimal classification or (2) irrelevant, when they do not contribute to the classification.

Multivariate pattern analyses were done using 120 EEG-extracted markers (corresponding to quantification of power spectrum and complexity in individual EEG sensors and information sharing between EEG sensors) as described in Sitt et al., 2014 (see supplementary material in the same paper) and 8 EKG extracted markers. We trained a Support Vector Classifier (SVC) to distinguish between the VS/UWS and the MCS patients with a penalization parameter (C) equal to 1 . The SVC was repeatedly cross-validated with randomized stratified $k$-folding $(k=8)$. Previously to the training of the classifier, relevant features were automatically selected keeping the highest $20 \%$ of the ANOVA F-value scores. Performance of the classifier was measured using AUC scores. We defined 3 sets of features: (1) EKG markers of cognitive processes, corresponding to the PRE and POST intervals for the Local and Global contrasts (termed EKG ${ }_{\mathrm{cog}}$ ), (2) EKG markers of baseline vegetative function (termed $E K G_{v e g}$ ) corresponding to the $\mathrm{HR}$ and $\mathrm{HRV}$ in the three frequencies previously defined and (3) EEG markers. We estimated the accuracy of the classification algorithm with 
6 different combinations of these sets of markers: (1) $E E G+E K G_{c o g}+E K G_{v e g}$, (2) EEG+EKG ${ }_{c o g}$, (3) $E E G+E K G_{v e g}$, (4) EEG markers only, (5) EEG with both $E K G_{\text {cog }}$ and $E K G_{\text {veg }}$ markers shuffled and (6) $E K G_{c o g}+E K G_{v e g}$ markers only. To minimize the effect of the random selection of folds, the AUC scores were averaged across 250 repetitions.

\section{Statistical Analysis}

Statistical analysis encompassed correlations using Pearson product-moment correlation coefficient ( $r$ ) and Spearman's rank correlation coefficient (rho) with corresponding $p$-values ( $p$ ). Pearson's chi-squared and Wilcoxon rank-sum test were used to test for independence between the diagnosis and the demographics information of the patients. Bayesian ANOVA were performed to test the differences between groups using the BayesFactor R package (JZS Bayes factor with “medium" default prior setting $r=0.5$; Morey, Rouder, \& Jamil, 2014; R Development Core Team, 2016; Rouder, Morey, Speckman, \& Province, 2012). Bayes Factors interpretation were done according to the Raftery scale ${ }^{34}$. Differences across stimulation blocks were tested using two-sided paired samples signed tests. MVPA models were tested using nonparametric Kruskal-Wallis test adjusted for multiple comparisons.

All these steps were performed with custom-made software made in Python 3.4 in combination with Scikit-Learn ${ }^{35}$ and MNE-Python ${ }^{36,37}$. Strengthening the Reporting of

Observational Studies in Epidemiology (STROBE) guidelines ${ }^{38}$ were followed throughout.

\section{(2) \\ Results \\ EKG extraction method validation}

To test the homogeneity of the EKG-related ICA decompositions between groups, we computed the mean IC weights across subjects for the selected components. A sensor-wise Bayesian t-test showed evidence for no difference in the weights between the MCS and VS/UWS groups (Figure $2 \mathrm{C}$ ). We then averaged the cardiac cycle locked to the QRS complex at the group level and the contrasted the obtained timeseries between clinical groups (Figure 2D). A single channel cluster permutation test found only one significant difference ( $p=0.017$ ) between 184 and $344 \mathrm{~ms}$ after the R-peak, consistent with the location of the T wave. No difference was found in the QRS complex. 
Finally, we aimed at ensuring that the results obtained in terms of phase shifts of the cardiac cycle induced by the processing of the auditory stimulation paradigm were not a side-effect of the EKG extraction methodology. In other words, we focused on testing that EEG-ICA extraction methodology was not injecting relevant EEG related activity to the EKG extracted signal. For this objective, we compared pure EKG to EEG extracted EKG. We performed simultaneous EEG-EKG recordings in an independent group of 24 healthy subjects and 32 patients (14 VS/UWS, $18 \mathrm{MCS}$ ). We applied the same EKG extraction method previously described and obtained 12 (50\%) healthy subjects and 12 (37.5\%; 3 VS/UWS; 9 MCS) patients with both direct EKG and indirect EEG-extracted EKG. We contrasted the two corresponding EKG timeseries in each trial, by subtracting the timing of the R-peaks in the direct EKG signal from EEG-extracted signal (Figure 2E). A repeated measures Bayesian ANOVA was computed using the $R_{E K G}-R_{E E G}$ time differences as the study variable; trial types (LSGS, LDGD, LDGS and LSGD) and clinical state as factors. All the models including the trial type as a factor presented positive evidence in favor of no difference $\left(\mathrm{BF}_{01}>=4.27\right)$.

Furthermore, the model that tested the interaction between clinical state and trial type presented even stronger evidence of no difference $\left(\mathrm{BF}_{01}>=15\right)$. Given that the only information used in this study was the timing of the R-peaks (automatically extracted and analyzed within subjects), the here presented validation results strongly suggest that no effect was induced by the adopted EKG extraction methodology.

\section{Baseline cardiac activity}

Overall Heart Rate (HR) was similar in patients across the two diagnostic groups $\left(B F_{10}=0.73\right.$; Figure $\left.3 A\right)$. When patients with overlapping behavioral CRS-R scores (CRS-R=6 or 7; 10 MCS; 20 VS/UWS) were excluded from the analysis there was evidence for faster heart frequencies in the VS/UWS group $\left(\mathrm{BF}_{10}=8.80\right)$. In the VS/UWS group a positive correlation was identified between the HR and the CRS-R scores ( $r h o=0.27, p=0.02$ ). No such correlation was found for the MCS patients. Similarly, HRV markers were comparable in both diagnostic groups (Figures 3B-D, HRV high frequencies $B F_{10}=0.62$; HRV low frequencies $B F_{10}=0.36, H R V$ very low frequencies $B_{10}=0.21$ ). In the VS/UWS group a positive correlation was identified between the CRS-R and the HRV markers in high frequencies (rho=0.40, p=0.0007) and in low frequencies ( $r h o=0.27, p=0.02$ ). No such correlations were identified for the MCS group in either frequency. 


\section{R-peak locked EEG evoked responses}

In terms of evoked responses to the cardiac activity as measured by EEG, a sharp peripheral bipolar topography was observed at the R-peak for both clinical groups (Figure 4A and B). Between 0 and $250 \mathrm{~ms}$ after the R-peak, both groups presented topographies following the pattern of the cardiac field artifact (CFA) ${ }^{26}$. A cluster-level permutation test revealed a single significant cluster ( $p=0.034$; Figure $4 C$ ) located between 144 and $540 \mathrm{~ms}$ after the R-peak, with two spatial patterns, one similar to the CFA associated to the T-wave between 144 and 340ms, and second central spatial pattern after 340ms.

\section{Cardiac cycle modulation induced by the auditory stimulation protocol}

There was no evidence for difference in cardiac cycle modulation between groups due to the processing of the Local regularities in either the PRE $\left(B_{10}=0.19\right)$ or the POST $\left(\mathrm{BF}_{10}=0.19\right)$ intervals (Figure 5). Within the groups, neither the VS/UWS nor the MCS patients presented significant differences between LS and LD trials (sign-test LD-LS trials, VS/UWS $p>0.7, \operatorname{MCS} p>0.2)$. In the case of the Global effect, there was no evidence of modulation difference between groups due to the global auditory processing in the PRE interval $\left(\mathrm{BF}_{10}=0.21\right)$. On the contrary, in the POST interval, there was a strong evidence for a difference between the MCS and VS/UWS groups $\left(\mathrm{BF}_{10}=43.07\right)$ (Figure 5$)$. This result is explained by a shortening of the POST intervals in the GD trials compared to the GS trials in the MCS patients (sign-test GD-GS trials, $p=0.007$ ) and no difference between GD and GS in the VS/UWS patients (sign-test GD-GS trials, $p=0.55$ ). The small sample of healthy controls $(n=12)$ included in this study presented a pattern of results similar to the MCS subjects (although not statistically significant).

\section{Correlation between EEG and EKG markers of consciousness}

We tested the relationship between cardiac cycle modulation markers and EEG markers that previously were reported to distinguishing VS/UWS and MCS patients ${ }^{7}$. These markers include quantifications of the spectral distribution of the EEG (such as power in the different frequency bands), the complexity of the EEG (such as Kolmogorov complexity and spectral entropy) and the information sharing between EEG electrodes (such as WSMI). The modulation of the POST interval due to the Global Effect significantly correlated with EEG Kolmogorov Complexity ( $K ; r=-2.31, p=0.02)$, Permutation Entropy ( $P E ; r=-2.63, p=0.01$ ), 
Spectral Entropy (SE; r=-2.3, p=0.02), Weighted Symbolic Mutual Information (wSMI; $r=-0.19$, $p=0.02)$ and normalized Delta Power $(r=0.2, p=0.02)$. No correlation was found between EEG evoked responses to the Global Effect and the phase shifts computed in the EKG (See table 1 for all markers). Nevertheless, none of the computed correlations survived a false discovery rate correction from multiple comparisons.

\section{Multivariate patient classification by means of EKG and EEG markers}

To determine if the EKG extracted information is partially independent to the consciousness related information extracted from the EEG, we trained classifiers to distinguish clinical groups and compared the performance of using as features EEG makers alone or combinations of EEG and EKG markers. Multivariate analysis combining EEG and $E G_{c o g}$ showed better performance compared to EEG and EKG ${ }_{\text {veg }}$ markers and EEG markers alone (Figure 6). Combining the $\mathrm{EKG}_{\mathrm{cog}}$ and EEG markers led to an improvement of the performance $\left(E E G+E K G_{c o g}, A U C=76.1 ; E E G+E K G_{c o g}+E K G_{v e g}, A U C=75.7\right)$. On the other hand, when $\mathrm{EKG}_{\mathrm{cog}}$ markers were not included in the MVPA the performance did not differ from EEG alone ( $E E G$ only, $A U C=73.7 ; E E G+E K G_{\text {veg }}, A U C=73.3$ ). As a control test for the effect of the number of features, classification was also computed combining EEG and label-shuffled EKG markers; in this case, the AUC was estimated at 73.6. Using solely cardiac markers, the classifier performed above chance with a mean AUC of 60.1. When we compared the performance of MVPAs that included EEG features, we only found significant differences when the MVPAs also included $\mathrm{EKG}_{\mathrm{cog}}$ versus when the MVPAs did not include these cardiac features ( $p<1 e-9$, Kruskall-Willis test, corrected for multiple comparisons). The inclusion of $E \mathrm{KG}_{\mathrm{veg}}$ features didn't significantly changed the performance of the tested MVPA classifiers $(p>0.1)$.

\section{Discussion}

We here aimed at characterizing consciousness state in patients with DOC by means of baseline heart activity and heart-brain interactions. We tested if cardiac-extracted information can complement single-patient EEG-based classification performance. When we contrasted behaviorally non-overlapping VS/UWS and MCS patients we found higher HR and HRV in the VS/UWS than MCS group, in accordance to a recent study ${ }^{39}$. This comparison included MCS patients who were in the higher end of the CRS-R scale versus the VS/UWS 
patients who were in the lower end of the CRS-R. When all DOC patients were included in order to retain clinical reality we did not find group differences of overall cardiac autonomic markers between the groups. This suggests a common underlying baseline cardiac function across patients. Interestingly, we found a positive correlation between CRS-R total scores and three autonomic markers (HR, HRV HF and HRF LF) only in the VS/UWS patients.

Our results are consistent with previous findings showing a relationship between the level of consciousness and dysautonomia in DOC after traumatic brain injuries. Specifically, low CRS-R scores were related to tachycardia in patients with low scores on the Glasgow Coma Scale ${ }^{20}$ and to lower HRV (in both high and low frequencies), which was considered as a symptom of a neurological disconnection syndrome ${ }^{21}$. Taken together, these results suggest that the diversity of behaviors characterizing conscious states (associated with cortical processing) does not necessarily translate into strong correlations with autonomic markers, such as HR and HRV. Therefore, the observed differences in these markers in VS/UWS patients on the lower end of the CRS-R scale seems to be associated with an overall deterioration of clinical condition, rather than to cognitive processing.

Our analysis of the heart evoked potentials revealed two results. First, we observed a statistical difference between VS/UWS and MCS in the CFA corresponding to the T wave but no difference in association to the QRS wave. The differences observed in the T-wave between VS/UWS and MCS patients, in the shape of a dipole with a left-posterior positivity and a right-frontal negativity, are similar to the reported cardiac repolarization changes induced by mental stress ${ }^{15}$ and neurodegeneration or stroke ${ }^{40}$. Although previous works depict a main modulation during the time window corresponding to the T-wave with frontal negativities, in our study the differences between the groups of DOC patients are highlighted by the cluster statistic in the posterior positive side of the dipole. Second, we found differences between VS/UWS and MCS patients in a time window after the T-wave. Crucially, this difference had a different topography to the previously described CFA. The maximal differences in the EEG were obtained in the central electrodes. Taken together these results further suggest differences in heart-brain interaction between VS/UWS and MCS patients. In terms of cognitive processing, we analyzed the cardiac activity while patients were evaluated with the 'Local Global' paradigm aiming to probe cognitive-related responses on cardiac markers. Such brain-heart interactions have been previously shown in protocols where, by quantifying neural events locked to heartbeats, one could predict whether a 
subject would report a fast flashing visual stimulus as perceived or not ${ }^{16}$. In addition, during complex cognitive processing, such as when playing chess, the heartrate dynamics, as measured before players made a move, could predict the likelihood of them eventually committing an error ${ }^{41}$. Heartbeat-evoked cortical responses were further shown to differ in auditory interoceptive learning tasks ${ }^{42}$ and emotional states ${ }^{43}$. Taken together, these studies suggest a bi-directional interaction between brain and heart that can be modulated by cognitive processes.

In our protocol, we found that the cardiac cycle was modulated by the processing of global auditory regularities only in the MCS group. Specifically, MCS patients showed an acceleration of the timing of the heartbeat following the auditory stimulus (shortening of the POST interval) which disrupted the global regularity. No such modulation of cardiac cycle was found in the VS/UWS patients, nor any effect was found in either group for the local irregularities. No modulations of the PRE intervals were found, this suggests that the only observed modulation is a direct effect of the cognitive process of the stimulation. It is important to compare this results with previous works that analyzed the evoked responses in the EEG using the same protocol. These studies show that the violations of local regularities (in the form of a mismatch negativity response) can be detected in healthy and awake controls but also unconscious conditions such as subjects during sleep, coma and VS/UWS ${ }^{44-46}$. In contrast, disruptions of the global regularities (eliciting a P3b response) are only present in conscious and attentive subjects (although see Tzovara et al. ${ }^{47}$ and Naccache et al. ${ }^{48}$ for ongoing discussions). The fact that cardiac cycle modulation effect was present only associated to global irregularities (which requires maintaining conscious attention) and only in the MCS patients only (who are generally characterized by more complex brain function compared to VS/UWS patients ${ }^{49}$ ) suggests that the source of this effect is a braindriven indirect modulation due to the conscious processing of information.

A recent study demonstrated a link between conscious perception and cardiac activity in normal subjects ${ }^{16}$. Specifically, in visual detection task, subjects' heart rate decreased during a warning cue and increased immediately after reporting the perception or not of the stimuli following the cue. When subjects responded correctly, following RR intervals were significantly shorter than the ones corresponding to an incorrect response. This indicates an interaction between conscious perception and the modulation of cardiac activity. Interestingly, previous studies showed that the characterization of the modulation depends 
on the stimulation inter-trial interval. With short intervals, this cardiac slowing is reversed within the same cycle that the target is detected ${ }^{50,51}$. In our work we depict a shortening of the RR interval containing the stimuli. Nevertheless, only when the stimulus is known to produce neural modulations and only in patients with higher level of consciousness. Our attention-driven effect is consistent with these previous results and characterizes the modulation in relation to the subjects' overall level of consciousness.

Having a proficient test at the single-subject level is a clinical necessity in order to reduce the diagnostic uncertainty each case. The modulation of the heart cycle within each subject was not powerful enough to have a significant effect to distinguish the clinical state of individual subject. With the aim of improving the single case performance of diagnostics tests, and particularly in terms of EEG, we have shown that multivariate classification performance of the combination of 120 EEG markers (such as quantifications comprising connectivity analysis, information complexity, spectral analysis and evoked related potentials) outperformed the univariate classification accuracy, when markers were considered individually. This combination of EEG markers allowed an enhanced classification of conscious state at single-patient level ${ }^{7}$. Although the cardiac measures alone did not allow a single subject diagnosis, combining information from both neural and cardiac sources increased significantly the accuracy of the classification of these patients. This indicates that the information extracted from the modulations of cardiac activity due to cognitive processing is partially independent from the neural correlates of consciousness as measured by EEG. To our knowledge, this is the first time that body-related signals are considered as contributing factors in data-driven diagnosis in patient with DOC. We think that such an embodied approach to cognition ${ }^{52}$ paves the way for further investigations of body-brain interactions in DOC which might be informative not only for clinics but also for tracing the neural correlates of consciousness. In the future, and with the aim of improving the single case performance of this test, we will introduce novel versions of stimulation paradigm (with stimulations contextually locked to the ongoing cardiac cycle).

In conclusion, we show a relation between autonomic nervous system function and a stimulation paradigm exposing subjects to violations of auditory regularities in MCS patients. Our results suggest that cardiac cycle modulation is relevant for the assessment of patients with DOC because it potentially carries partially independent information when taken together with neural correlates of consciousness. We think that our work opens a window to 
the study of DOC via the embodied paradigm, according to which body-brain functions contribute to a holistic approach to conscious processing.

\section{Acknowledgments}

This work was supported by Consejo Nacional de Investigaciones Científicas y Técnicas (Argentina), Institut National de la Santé et de la Recherche Médicale (France), the James S. McDonnell Foundation and the Institut du Cerveau et de la Moelle Épinière (France), the FRM Equipe 2015 grant, and the Lamonica Prize from the Académie des Sciences. STICAmSud grants COMPLEX and RTBRAIN. The authors wish to thank Dr Catherine TallonBaudry for reviewing a preliminary version of this work and Dr Sophie Demeret for addressing us some of the patients recorded in this study. This study is dedicated to the patients and to their close relatives.

\section{Author Contributions}

F.R., M.S., D.F.S., L.N., and J.S. contributed to the conception and design of the study; F.R.,

B.R., M.V., and J.S. contributed to the acquisition and analysis of data; F.R., A.D., M.S., D.E., L.N. and J.S., contributed to the drafting of the manuscript and/or figures.

\section{Potential Conflicts of Interest}

Nothing to report

\section{References}

1. Laureys S, Owen AM, Schiff ND. Brain function in coma, vegetative state, and related disorders. Lancet Neurol. 2004;3(9):537-546.

2. Laureys S, Celesia GG, Cohadon F, et al. Unresponsive wakefulness syndrome: a new name for the vegetative state or apallic syndrome. BMC Med. 2010;8(1):68.

3. Rosenberg N, Whyte J, Zafonte RD, et al. The minimally conscious state Definition and diagnostic criteria. Neurology 2002;58(3):349-353.

4. Giacino JT, Kalmar K, Whyte J. The JFK Coma Recovery Scale-Revised: Measurement characteristics and diagnostic utility. Arch. Phys. Med. Rehabil. 2004;85(12):20202029.

5. Schnakers C, Vanhaudenhuyse A, Giacino J, et al. Diagnostic accuracy of the vegetative and minimally conscious state: clinical consensus versus standardized neurobehavioral assessment. BMC Neurol. 2009;9:35. 
6. Rohaut B, Faugeras F, Naccache L. Neurology of consciousness impairments. Brain Disord. Crit. Illn. Mech. Diagnosis, Treat. 2013;

7. Sitt JD, King JR, El Karoui I, et al. Large scale screening of neural signatures of consciousness in patients in a vegetative or minimally conscious state. Brain 2014;137(8):2258-2270.

8. Demertzi A, Antonopoulos G, Heine L, et al. Intrinsic functional connectivity differentiates minimally conscious from unresponsive patients. Brain 2015;138(9):2619-2631.

9. Casarotto S, Comanducci A, Rosanova M, et al. Stratification of unresponsive patients by an independently validated index of brain complexity. Ann. Neurol. 2016;

10. Stender J, Mortensen KNN, Thibaut A, et al. The Minimal Energetic Requirement of Sustained Awareness after Brain Injury. 2016.

11. Luyt C-E, Galanaud D, Perlbarg V, et al. Diffusion tensor imaging to predict long-term outcome after cardiac arrest: a bicentric pilot study. Anesthesiology 2012;117(6):1311-21.

12. Critchley HD, Mathias CJ, Dolan RJ. Neural Activity in the Human Brain Relating to Uncertainty and Arousal during Anticipation. Neuron 2001;29(2):537-545.

13. Craig AD. How do you feel? Interoception: the sense of the physiological condition of the body. Nat. Rev. Neurosci. 2002;3(8):655-666.

14. Critchley HD, Harrison NA. Visceral Influences on Brain and Behavior. Neuron 2013;77(4):624-638.

15. Gray MA, Taggart $P$, Sutton $P M$, et al. A cortical potential reflecting cardiac function. [date unknown];

16. Park H-D, Correia S, Ducorps A, Tallon-Baudry C. Spontaneous fluctuations in neural responses to heartbeats predict visual detection. Nat. Neurosci. 2014;17(4):612-8.

17. Seth AK, Suzuki K, Critchley HD. An interoceptive predictive coding model of conscious presence. Front. Psychol. 2012;3(JAN)

18. Park H-D, Tallon-Baudry C, Koch C, et al. The neural subjective frame: from bodily signals to perceptual consciousness. Philos. Trans. R. Soc. Lond. B. Biol. Sci. 2014;369(1641):20130208.

19. Lacey BC, Lacey Jl. Two-way communication between the heart and the brain. Significance of time within the cardiac cycle. Am. Psychol. 1978;33(2):99-113.

20. Baguley IJ, Nicholls JL, Felmingham KL, et al. Dysautonomia after traumatic brain injury: a forgotten syndrome? J. Neurol. Neurosurg. Psychiatry 1999;67(1):39-43.

21. Baguley IJ, Heriseanu RE, Felmingham KL, Cameron ID. Dysautonomia and heart rate variability following severe traumatic brain injury. Brain Inj. 2006;20(4):437-444.

22. Bekinschtein $T A$, Dehaene $S$, Rohaut $B$, et al. Neural signature of the conscious processing of auditory regularities. Proc. Natl. Acad. Sci. 2009;106(5):1672-1677.

23. Hyvärinen A, Oja E. Independent component analysis: Algorithms and applications. Neural Networks 2000;13(4-5):411-430.

24. Bell AJ, Sejnowski TJ. An information-maximization approach to blind separation and blind deconvolution. Neural Comput. 1995;7(6):1129-1159.

25. Lee TW, Girolami M, Sejnowski TJ. Independent component analysis using an extended infomax algorithm for mixed subgaussian and supergaussian sources. Neural Comput. 1999;11:417-441.

26. Dirlich G, VogI L, Plaschke M, Strian F. Cardiac field effects on the EEG. 1997;102:307315. 
27. Elgendi M. Fast QRS Detection with an Optimized Knowledge-Based Method: Evaluation on 11 Standard ECG Databases. PLoS One 2013;8(9)

28. Deboer RW, Karemaker JM, Strackee J. Comparing Spectra of a Series of Point Events Particularly for Heart Rate Variability Data. IEEE Trans. Biomed. Eng. 1984;BME31(4):384-387.

29. Maris E, Oostenveld R. Nonparametric statistical testing of EEG- and MEG-data. 2007;164:177-190.

30. Kohavi R, John GH. Wrappers for feature subset selection. Artif. Intell. 1997;97(12):273-324.

31. Rouder JN, Morey RD, Speckman PL, Province JM. Default Bayes factors for ANOVA designs. J. Math. Psychol. 2012;56(5):356-374.

32. Morey RD, Rouder JN, Jamil T. BayesFactor: Computation of Bayes factors for common designs. R Packag. version 0.9 2014;8

33. R Development Core Team. R: A Language and Environment for Statistical Computing. R Found. Stat. Comput. Vienna Austria 2016;0:\{ISBN\} 3-900051-07-0.

34. Kass R, Raftery A. Bayes Factors. J. Amer. Stat. Assoc. 1995;90(430):773-795.

35. Pedregosa F, Varoquaux G, Gramfort A, et al. Scikit-learn: Machine Learning in Python. J. Mach. Learn. Res. 2012;12:2825-2830.

36. Gramfort A, Luessi M, Larson E, et al. MNE software for processing MEG and EEG data. Neuroimage 2014;86:446-460.

37. Gramfort A, Luessi M, Larson E, et al. MEG and EEG data analysis with MNE-Python. Front. Neurosci. 2013;7(7 DEC):267.

38. von Elm E, Altman DG, Egger $M$, et al. The strengthening the reporting of observational studies in epidemiology (STROBE) statement: Guidelines for reporting observational studies. Int. J. Surg. 2014;12(12):1495-1499.

39. Leo A, Naro A, Cannavò A, et al. Could autonomic system assessment be helpful in disorders of consciousness diagnosis? A neurophysiological study. Exp. Brain Res. 2016;234(8):2189-2199.

40. García-Cordero I, Sedeño L, De La Fuente L, et al. Feeling, learning from, and being aware of inner states: Interoceptive dimensions in neurodegeneration and stroke. Philos. Trans. R. Soc. London B Biol. Sci. 2016;(Accepted):0-34.

41. Leone MJ, Petroni A, Fernandez Slezak D, Sigman M. The tell-tale heart: heart rate fluctuations index objective and subjective events during a game of chess. Front. Hum. Neurosci. 2012;6(October):273.

42. Canales-Johnson A, Silva C, Huepe $D$, et al. Auditory feedback differentially modulates behavioral and neural markers of objective and subjective performance when tapping to your heartbeat. Cereb. Cortex 2015;25(11):4490-4503.

43. Couto $B$, Adolfi $F$, Velasquez $M$, et al. Heart evoked potential triggers brain responses to natural affective scenes: A preliminary study. Auton. Neurosci. Basic Clin. 2015;193:132-137.

44. Faugeras $\mathrm{F}$, Rohaut $\mathrm{B}$, Weiss $\mathrm{N}$, et al. Probing consciousness with event-related potentials in the vegetative state. Neurology 2011;77(3):264-268.

45. Strauss M, Sitt JD, King J-R, et al. Disruption of hierarchical predictive coding during sleep. Proc. Natl. Acad. Sci. 2015; In press.

46. Morlet D, Fischer C. MMN and Novelty P3 in Coma and Other Altered States of Consciousness: A Review. Brain Topogr. 2013;

47. Tzovara A, Simonin A, Oddo M, et al. Neural detection of complex sound sequences in 
the absence of consciousness. Brain 2015;138(5):1160-1166.

48. Naccache L, King J-R, Sitt J, et al. Neural detection of complex sound sequences in the absence of consciousness? Brain 2015;138

49. Giacino JT, Fins JJ, Laureys S, Schiff ND. Disorders of consciousness after acquired brain injury: the state of the science. Nat. Rev. Neurol. 2014;10(2):99-114.

50. Van Der Molen MW, Somsen RJM, Orlebeke JF. Phasic heart rate responses and cardiac cycle time in auditory choice reaction time. Biol. Psychol. 1983;16(3-4):255271.

51. Jennings JR, Wood CC. Cardiac Cycle Time Effects on Performance, Phasic Cardiac Responses, and Their Intercorrelation in Choice Reaction Time. Psychophysiology 1977;14(3):297-307.

52. Clark A. An embodied cognitive science? Trends Cogn. Sci. 1999;3(9):345-351.

\section{Figure Legends}

Figure 1.

\section{Illustration of the auditory paradigm (A) and the cardiac-related markers used in the} analysis (B).

(A) Each trial of the auditory paradigm was composed by 5 consecutive sounds. Four equal sounds defined a local regularity (music notes). The $5^{\text {th }}$ sound could be equal or different, defining a local standard or deviant trial respectively. At a second level, frequent trials (80\%, left shaded area) defined a global regularity and rare trials (20\%, right shaded area) violated this regularity. (B) Two consecutive EKG QRS complexes defined an RR interval. Overall heart rate was computed from the robust average of the RR intervals. Conscious response to the stimulation was analyzed based on the definition of two intervals: (1) the PRE interval, measured between the R-peak previous to the $5^{\text {th }}$ sound and the onset of that sound and (2) the POST interval, measured between the onset of the $5^{\text {th }}$ sound and the following R-peak. In order to avoid coupling with the heartbeats, trials in which the sound was less than $20 \mathrm{~ms}$ or more than 600 ms apart from the R-peak were discarded.

\section{Figure 2.}

EKG Independent components present no difference in the QRS complex between clinical groups.

(A) Timeseries from 7 typical EEG sensors from one UWS patient. (B) Corresponding timeseries of 7 ICA components extracted from the previous EEG recording and the respective weights topographies. The independent component with cardiac information is 
shown in red. Dotted lines represents the automatically detected R-peak. (C) Mean weights topographies for each clinical group (top). A sensor-wise Bayesian t-test shows evidence for no difference in the topographies between groups (bottom). (D) Mean and standard error of the mean for each clinical group QRS complex from the ICA-extracted EKG. A single channel cluster permutation test indicated significant differences $(p=0.017)$ only between 184 and $344 \mathrm{~ms}$ after the R-peak, consistent with the location of the T wave. (E) We evaluated two independent groups of healthy controls $(n=12)$ and patients $(n=12)$ using simultaneous EEG and EKG recordings. For each subject, EKG was also extracted using the described ICA method. We then computed the differences between each R-peak onset detected in the direct EKG and the corresponding R-peak detected using ICA (left). Right panel shows the mean difference and $95 \% \mathrm{Cl}$ for each type of trial and subject as measured in samples (1 sample $=4 \mathrm{~ms}$ ). Using Bayesian ANOVA, we found no evidence for a difference as an effect of the trial type $\left(\mathrm{BF}_{01}>4.27\right)$ and strong evidence for no difference for the interaction between the type of trial and the clinical state $\left(\mathrm{BF}_{01}>15\right)$.

\section{Figure 3.}

\section{Cardiac autonomic markers show no difference between clinical groups. Lower CRS-R} scores in VS/UWS patients correlates with a faster and less variable cardiac cycle as a manifestation of the overall deterioration of the clinical condition.

Each panel depicts the cardiac marker values ( $y$-axis, Heart rate $(A)$, Heart rate variability in high frequencies (B), in low frequencies (C), and in very low frequencies (D)) for each patient (dot), categorized by clinical group (top, 70 VS/UWS, 57 MCS, 12 Healthy) and by Coma Recovery Scale-Revised scores (CRS-R, bottom - only for patients). The Spearman's regression line between the scores and the EKG-related markers for the VS/UWS patients, indicates a significant positive correlation between the CRS-R score and Heart Rate (A), Heart Rate Variability in high frequencies (B), and in low frequencies (C). Boxplots with interquartile range, median (black line) and mean (dashed line) represent the distribution of data in both clinical groups.

\section{Figure 4.}

\section{R-peak locked EEG evoked responses shows differences between clinical groups}


(A) Mean EEG topographies for each clinical group time locked to the R-peak at 0, 100, 250, 350,450 and $500 \mathrm{~ms}$. (B) Contrast and statistical comparison of the R-peak evoked potentials between clinical groups. (C) The left panel shows the only significant cluster of a permutation analysis $(p=0.034)$, the electrodes composing the cluster are shown with white circles (left). The right panel shows the timeseries of the corresponding cluster (mean and standard deviation across subjects). Two main modulations are observed, (1) in the time window corresponding to the T wave, a left-posterior positivity and a right-frontal negativity ; and, (2) a central electrode spatial pattern positivity after the T-wave (>350ms), suggesting differences in the brain processing of the heart activity between VS/UWS and MCS patients.

Figure 5.

Violations of global regularities induce cardiac cycle phase acceleration only in minimally conscious patients.

Local violations did not affect the ongoing cardiac activity for the intervals between the stimulation onset and the preceding R-peak (PRE, top left) nor the following R-peak (POST, top right). Similarly, global violations did not affect the ongoing cardiac activity at the PRE interval (bottom left). In clear contrast, they induced shortened POST intervals (bottom right) only in the minimally conscious state (MCS) group (between-group contrast BF=34.15; within-group sign-test $p$-value=0.01). The small sample of healthy controls included in this study presented a pattern of results similar to the MCS subjects (although not statistically significant). Each dot represents a patient in vegetative state/unresponsive wakefulness syndrome (VS/UWS, $\mathrm{N}=70$ ), in minimally consciousness state (MCS, $\mathrm{N}=57$ ) or a healthy control (Healthy, $n=12$ ). Boxplots with interquartile range, median (black line) and mean (dashed line) represent the distribution of data in the clinical groups.

Figure 6.

\section{Cognitive EKG markers carries partially independent information from EEG.}

Six distinct multivariate classifiers were trained to distinguish between VS/UWS and MCS patients using different combinations of EKG and EEG markers. We used as features

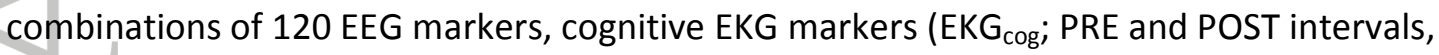

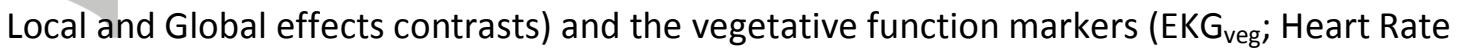
and Variability). All the models that summed EEG markers and $\mathrm{EKG}_{\mathrm{cog}}$, presented a significant 
increase in the classification accuracy (compared to MVPA of EEG without $E_{\text {cog }}, p<1 e-9$ ).

Using only EEG markers (mean AUC 73.7) showed no significant difference with EEG in combination with $\mathrm{EKG}_{\mathrm{veg}}$ markers (mean AUC 73.3). As a control to equalize the number of features, the combination of EEG and all of the EKG markers with shuffled labels reported a mean AUC of 73.3. When we used only EKG markers, the classifier performed above chance, obtaining a mean AUC of 60.1. Means were estimated using 250 repetitions of stratified 8fold cross validation. Each dot represents the mean value across folds for each repetition. Boxplots with interquartile range, median and mean (dotted line) represent the distribution of values for each set of features.

\section{Tables}

MARKER

R STATISTic P-VAlue P-VAlue (FDR CORRECTED)

\begin{tabular}{|l|lll|}
\hline CNV & 0.45 & 0.649 & 0.744 \\
\hline K & -2.31 & $0.022 *$ & 0.138 \\
\hline PE & -2.63 & $0.009 *$ & 0.138 \\
\hline ALPHA & -1.30 & 0.192 & 0.412 \\
\hline ALPHA N & -1.31 & 0.190 & 0.412 \\
BETA & -1.91 & 0.058 & 0.251 \\
\hline BETA N & -1.53 & 0.128 & 0.349 \\
\hline DELTA & 0.85 & 0.393 & 0.562 \\
\hline DELTA N & 2.40 & $0.017 *$ & 0.138 \\
\hline GAMMA & -1.67 & 0.096 & 0.321 \\
\hline GAMMA N & -1.43 & 0.154 & 0.385 \\
\hline SE & -2.30 & $0.023 *$ & 0.138 \\
\hline THETA & -0.50 & 0.616 & 0.744 \\
\hline THETA N & -0.94 & 0.345 & 0.562 \\
MSF & -1.61 & 0.108 & 0.324 \\
\hline SEF90 & -1.87 & 0.062 & 0.251 \\
\hline SEF95 & -1.84 & 0.067 & 0.251 \\
\hline WSMI & -2.34 & $0.020 *$ & 0.138 \\
\hline GD-GS & 0.54 & 0.589 & 0.744 \\
\hline LD-LS & 0.43 & 0.662 & 0.744 \\
\hline LSGD-LDGS & -0.31 & 0.756 & 0.810 \\
\hline LSGS-LDGD & -0.99 & 0.320 & 0.562 \\
\hline MMN & -0.86 & 0.387 & 0.562 \\
\hline DELTA P3A & -0.02 & 0.979 & 0.979 \\
\hline DELTA P3B & -0.42 & 0.669 & 0.744 \\
\hline P1 & -0.22 & 0.820 & 0.848 \\
\hline TOPO P3A & -0.86 & 0.387 & 0.562 \\
\hline
\end{tabular}




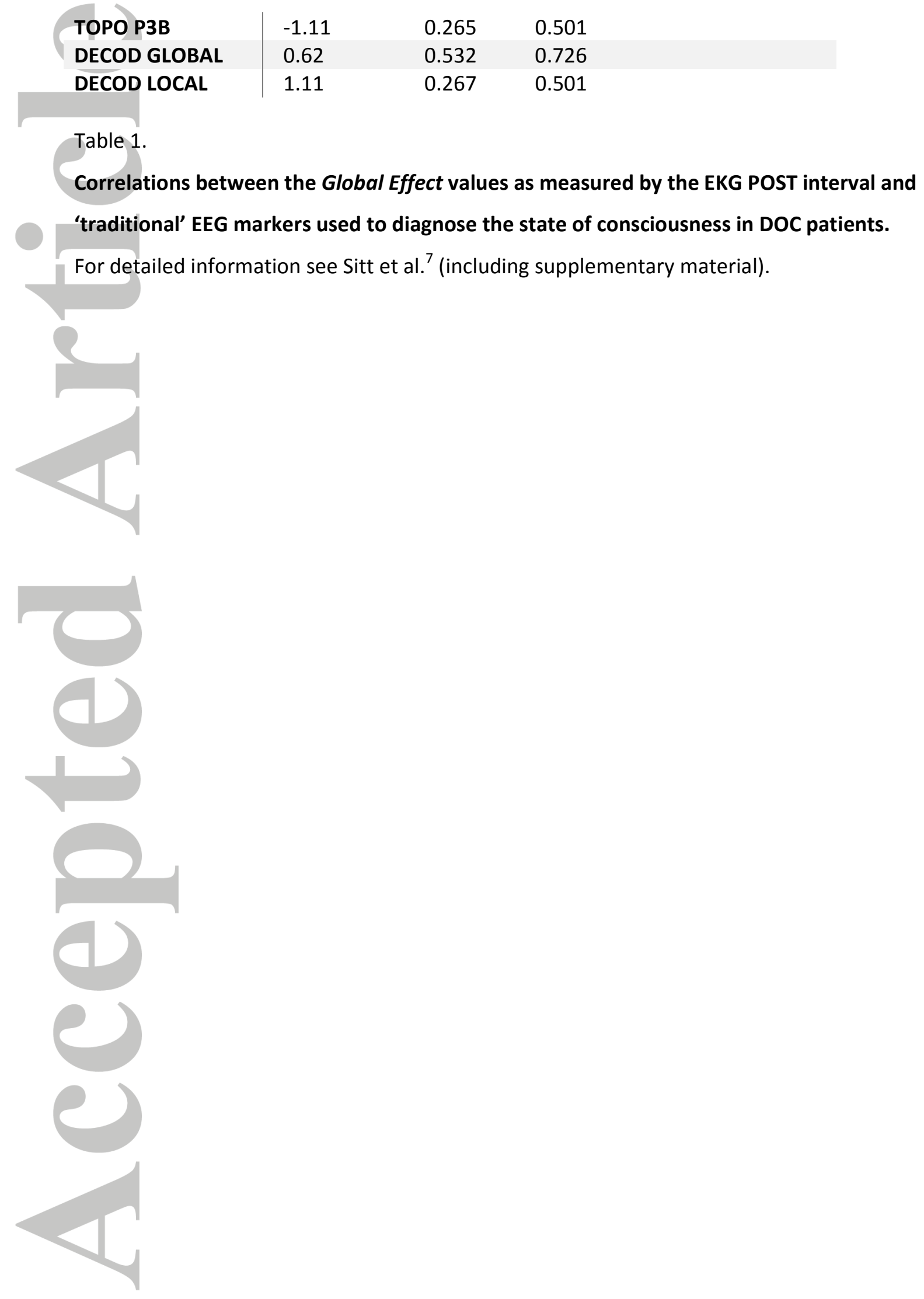


(A)

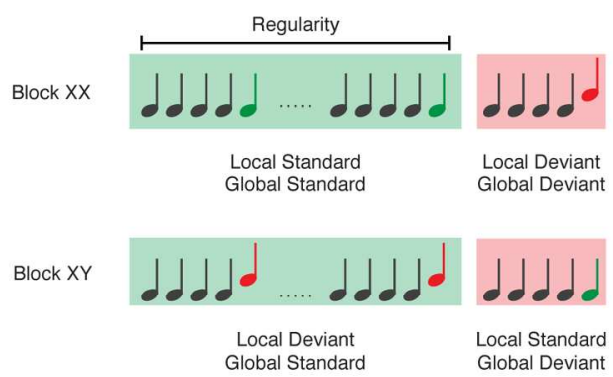

(B)

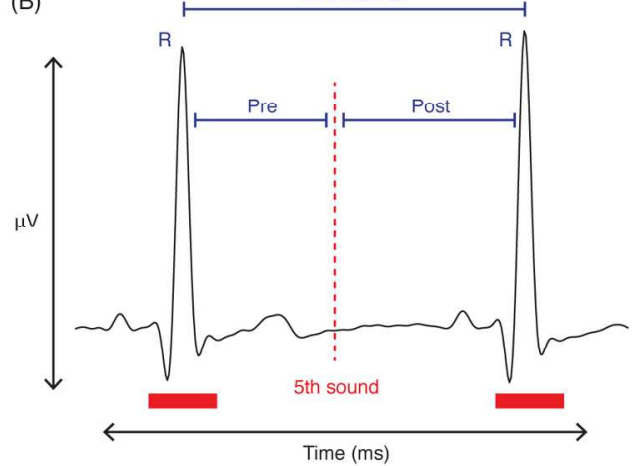

Illustration of the auditory paradigm (A) and the cardiac-related markers used in the analysis (B). (A) Each trial of the auditory paradigm was composed by 5 consecutive sounds. Four equal sounds defined a local regularity (music notes). The 5th sound could be equal or different, defining a local standard or deviant trial respectively. At a second level, frequent trials ( $80 \%$, left shaded area) defined a global regularity and rare trials ( $20 \%$, right shaded area) violated this regularity. (B) Two consecutive EKG QRS complexes defined an RR interval. Overall heart rate was computed from the robust average of the RR intervals. Conscious response to the stimulation was analyzed based on the definition of two intervals: (1) the PRE interval, measured between the R-peak previous to the 5th sound and the onset of that sound and (2) the POST interval, measured between the onset of the 5th sound and the following R-peak. In order to avoid coupling with the heartbeats, trials in which the sound was less than $20 \mathrm{~ms}$ or more than $600 \mathrm{~ms}$ apart from the R-peak were discarded.

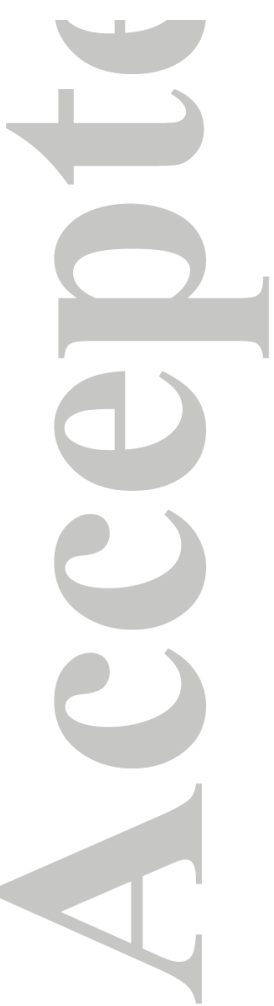

$167 \times 62 \mathrm{~mm}(300 \times 300$ DPI $)$ 
(A) Electroencephalogram

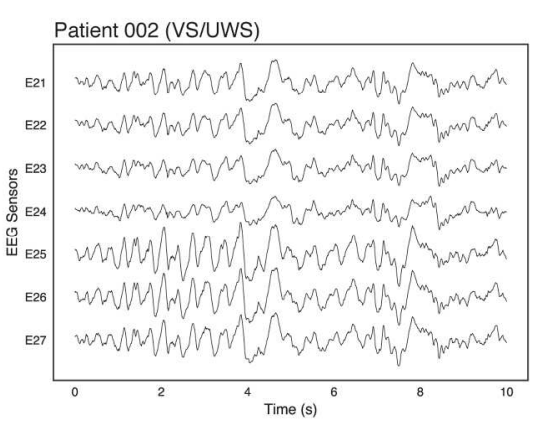

(C) Group IC Topographies
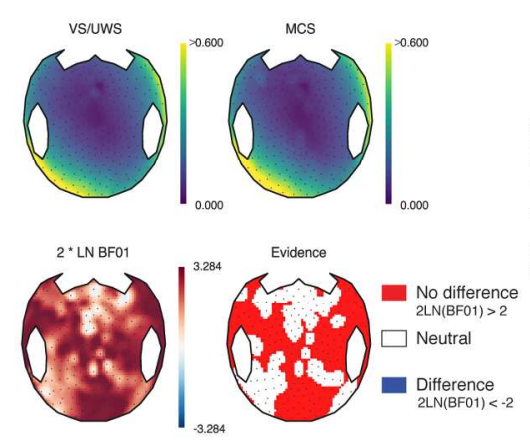

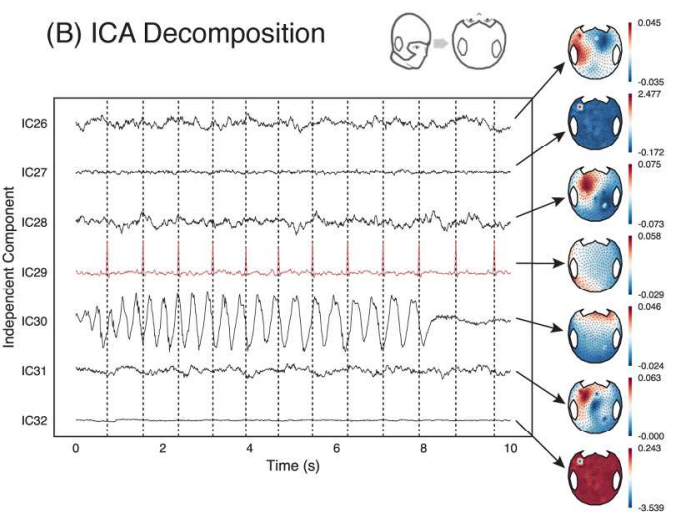

(D) Group EKG

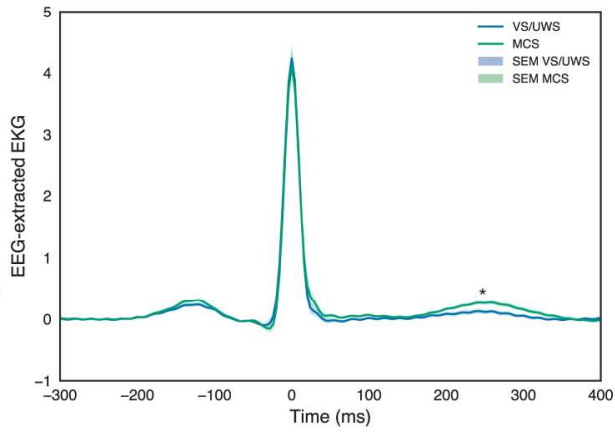

(E) Direct vs ICA-Extracted EKG
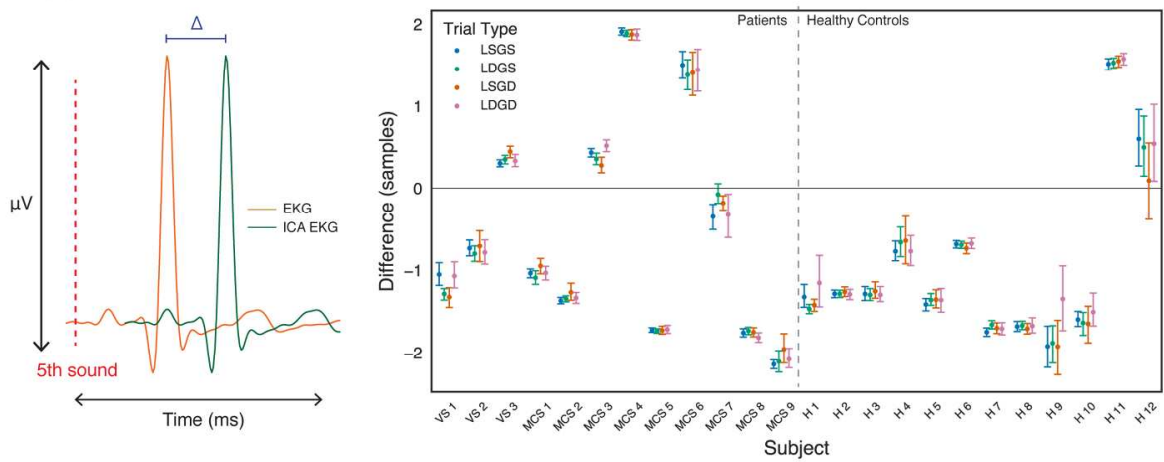

EKG Independent components present no difference in the QRS complex between clinical groups. (A) Timeseries from 7 typical EEG sensors from one UWS patient. (B) Corresponding timeseries of 7 ICA components extracted from the previous EEG recording and the respective weights topographies. The independent component with cardiac information is shown in red. Dotted lines represents the automatically detected R-peak. (C) Mean weights topographies for each clinical group (top). A sensor-wise Bayesian t-test shows evidence for no difference in the topographies between groups (bottom). (D) Mean and standard error of the mean for each clinical group QRS complex from the ICA-extracted EKG. A single channel cluster permutation test indicated significant differences $(p=0.017)$ only between 184 and $344 \mathrm{~ms}$ after the R-peak, consistent with the location of the $T$ wave. (E) We evaluated two independent groups of healthy controls $(n=12)$ and patients $(n=12)$ using simultaneous EEG and EKG recordings. For each subject, EKG was also extracted using the described ICA method. We then computed the differences between each R-peak onset detected in the direct EKG and the corresponding R-peak detected using ICA (left). Right panel shows the mean difference and $95 \% \mathrm{CI}$ for each type of trial and subject as measured in samples ( 1 sample $=4 \mathrm{~ms}$ ).

\section{John Wiley \& Sons}

This article is protected by copyright. All rights reserved. 
Using Bayesian ANOVA, we found no evidence for a difference as an effect of the trial type (BF01>4.27) and strong evidence for no difference for the interaction between the type of trial and the clinical state (BF01>15).

$178 \times 225 \mathrm{~mm}(300 \times 300 \mathrm{DPI})$
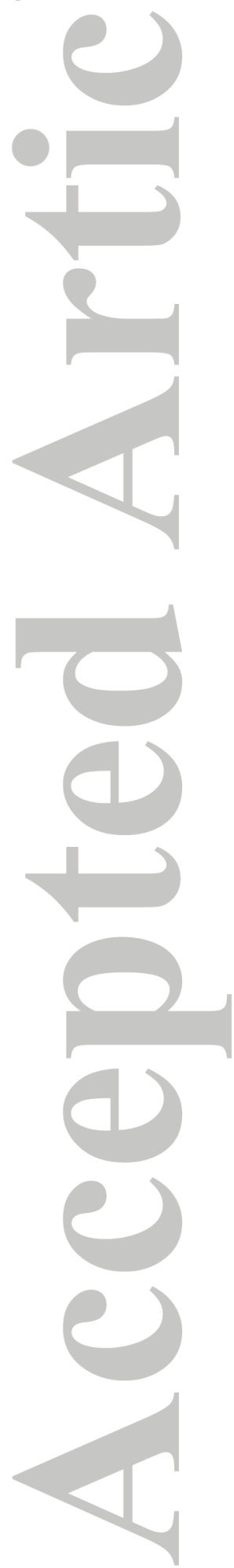

John Wiley \& Sons

This article is protected by copyright. All rights reserved. 
(A) Heart Rate

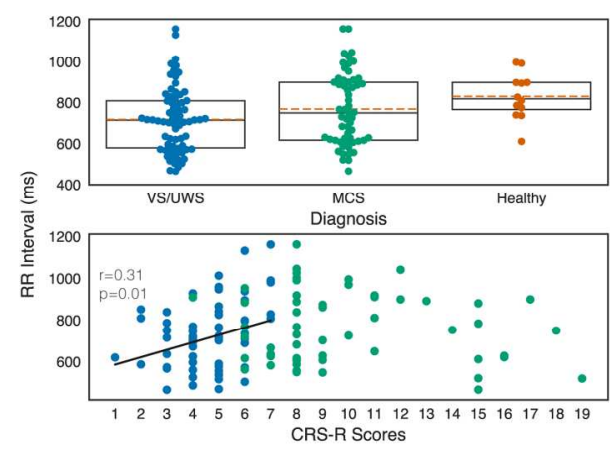

(C) Heart Rate Variability (Low Frequencies)

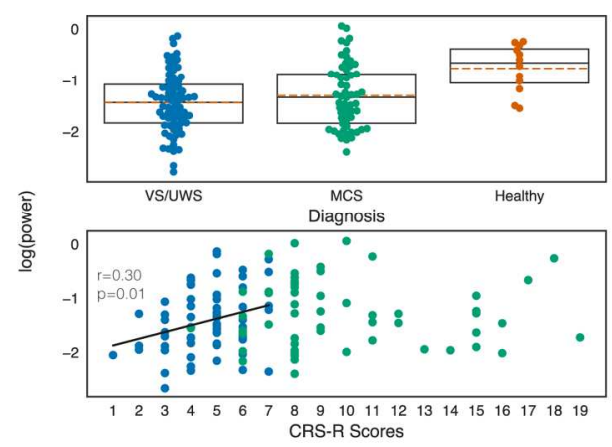

(B) Heart Rate Variability (High Frequencies)

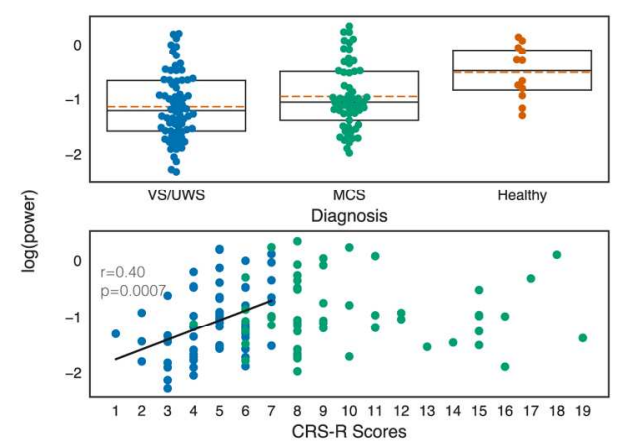

(D) Heart Rate Variability (Very Low Frequencies)

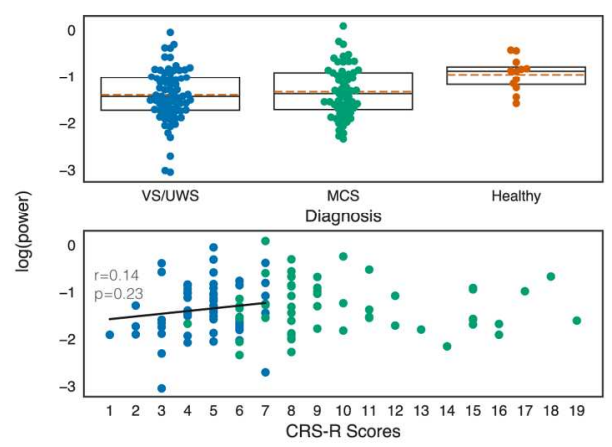

- Cardiac autonomic markers show no difference between clinical groups. Lower CRS-R scores in VS/UWS patients correlates with a faster and less variable cardiac cycle as a manifestation of the overall deterioration of the clinical condition.

Each panel depicts the cardiac marker values ( $y$-axis, Heart rate $(A)$, Heart rate variability in high frequencies (B), in low frequencies (C), and in very low frequencies (D)) for each patient (dot), categorized by clinical group (top, 70 VS/UWS, 57 MCS, 12 Healthy) and by Coma Recovery Scale-Revised scores (CRS-

$\mathrm{R}$, bottom - only for patients). The Spearman's regression line between the scores and the EKG-related markers for the VS/UWS patients, indicates a significant positive correlation between the CRS-R score and Heart Rate (A), Heart Rate Variability in high frequencies (B), and in low frequencies (C). Boxplots with interquartile range, median (black line) and mean (dashed line) represent the distribution of data in both clinical groups.

$170 \times 141 \mathrm{~mm}(300 \times 300 \mathrm{DPI})$

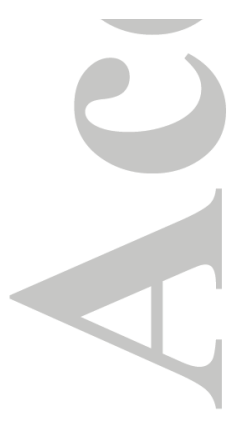


(A) R-Peak locked Evoked Responses

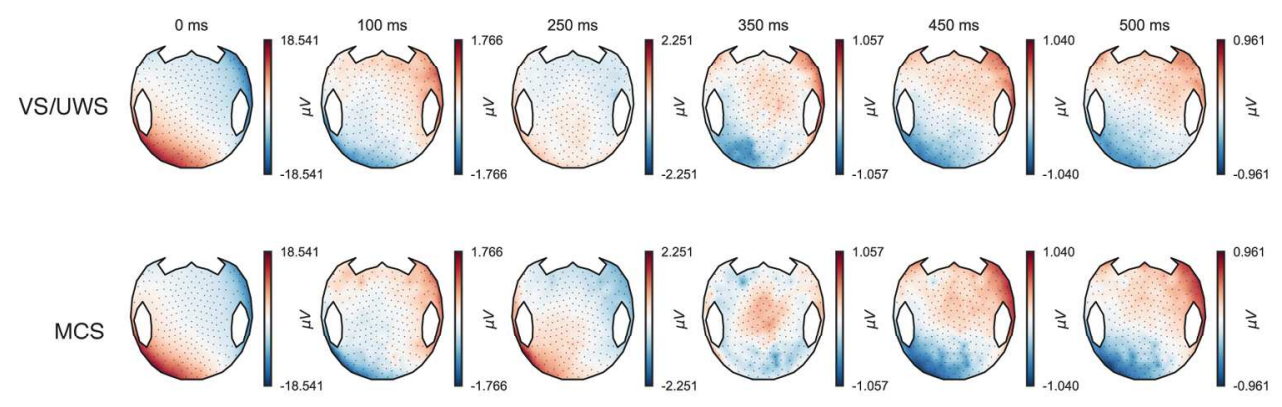

(B) MCS - VS/UWS Contrast
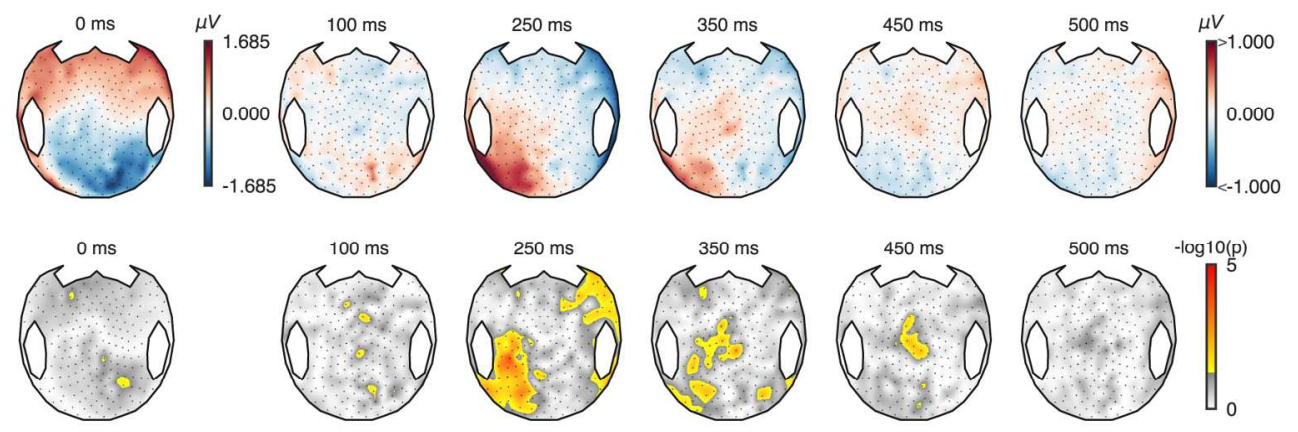

(C) Cluster analysis
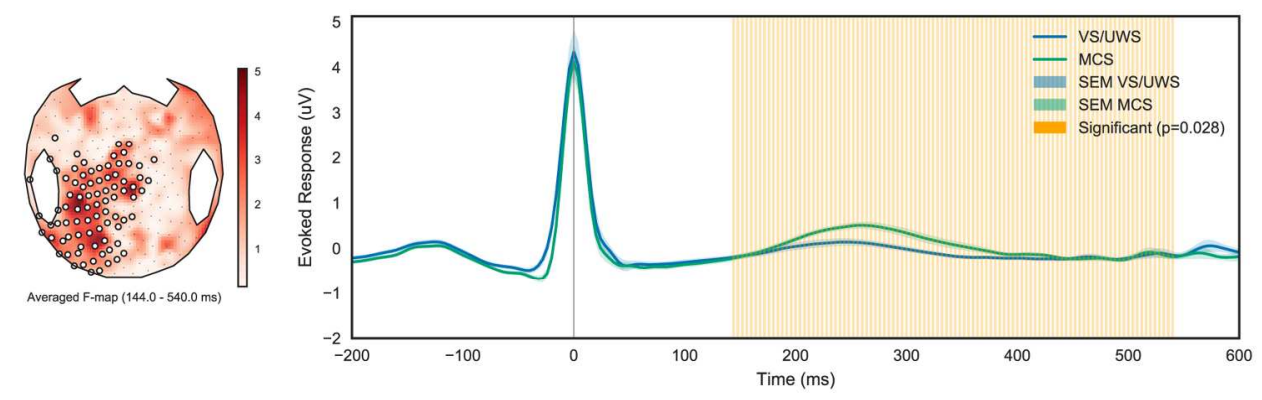

R-peak locked EEG evoked responses shows differences between clinical groups

(A) Mean EEG topographies for each clinical group time locked to the R-peak at 0, 100,250, 350, 450 and 500 ms. (B) Contrast and statistical comparison of the R-peak evoked potentials between clinical groups. (C) The left panel shows the only significant cluster of a permutation analysis $(p=0.034)$, the electrodes composing the cluster are shown with white circles (left). The right panel shows the timeseries of the corresponding cluster (mean and standard deviation across subjects). Two main modulations are observed, (1) in the time window corresponding to the T wave, a left-posterior positivity and a right-frontal negativity ; and, (2) a central electrode spatial pattern positivity after the T-wave (>350ms), suggesting differences in the brain processing of the heart activity between VS/UWS and MCS patients.

$170 \times 193 \mathrm{~mm}(300 \times 300 \mathrm{DPI})$ 

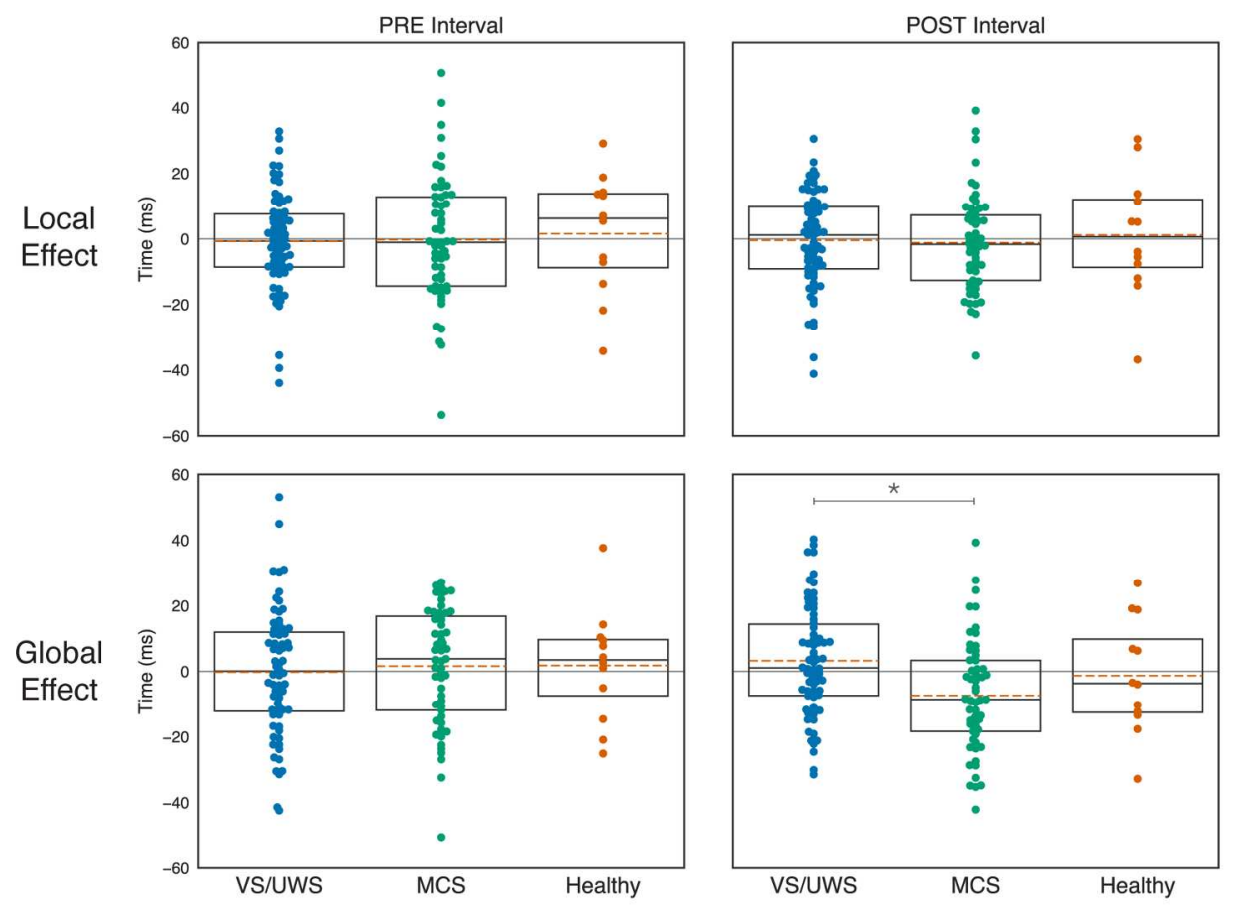

Violations of global regularities induce cardiac cycle phase acceleration only in minimally conscious patients. Local violations did not affect the ongoing cardiac activity for the intervals between the stimulation onset and the preceding R-peak (PRE, top left) nor the following R-peak (POST, top right). Similarly, global violations did not affect the ongoing cardiac activity at the PRE interval (bottom left). In clear contrast, they induced shortened POST intervals (bottom right) only in the minimally conscious state (MCS) group (between-group contrast $B F=34.15$; within-group sign-test $p$-value $=0.01$ ). The small sample of healthy controls included in this study presented a pattern of results similar to the MCS subjects (although not statistically significant). Each dot represents a patient in vegetative state/unresponsive wakefulness syndrome (VS/UWS, $N=70$ ), in minimally consciousness state (MCS, N=57) or a healthy control (Healthy, $\mathrm{n}=12$ ). Boxplots with interquartile range, median (black line) and mean (dashed line) represent the distribution of data in the clinical groups.

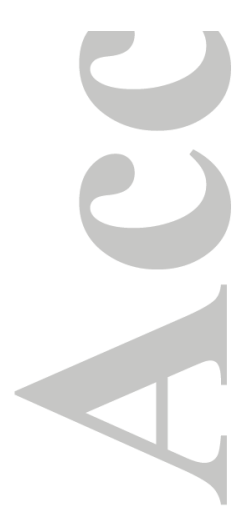

$170 \times 130 \mathrm{~mm}(300 \times 300 \mathrm{DPI})$ 


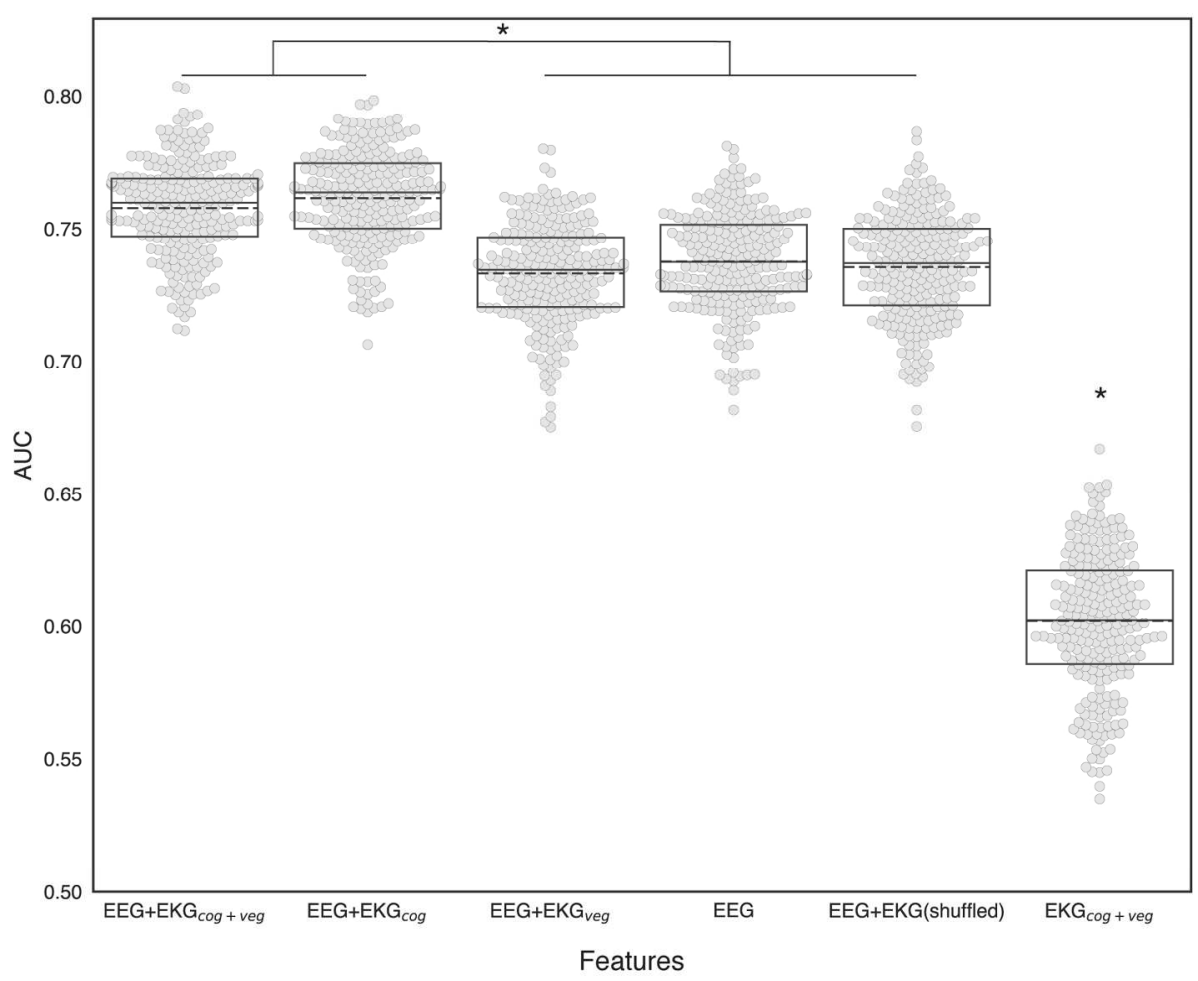

Cognitive EKG markers carries partially independent information from EEG.

Six distinct multivariate classifiers were trained to distinguish between VS/UWS and MCS patients using different combinations of EKG and EEG markers. We used as features combinations of 120 EEG markers, cognitive EKG markers (EKGcog; PRE and POST intervals, Local and Global effects contrasts) and the vegetative function markers (EKGveg; Heart Rate and Variability). All the models that summed EEG markers and EKGcog, presented a significant increase in the classification accuracy (compared to MVPA of EEG without EKGcog, $\mathrm{p}<1 \mathrm{e}-9$ ). Using only EEG markers (mean AUC 73.7) showed no significant difference with EEG in combination with EKGveg markers (mean AUC 73.3). As a control to equalize the number of features, the combination of EEG and all of the EKG markers with shuffled labels reported a mean AUC of 73.3. When we used only EKG markers, the classifier performed above chance, obtaining a mean AUC of 60.1. Means were estimated using 250 repetitions of stratified 8 -fold cross validation. Each dot represents the mean value across folds for each repetition. Boxplots with interquartile range, median and mean (dotted line) represent the distribution of values for each set of features.

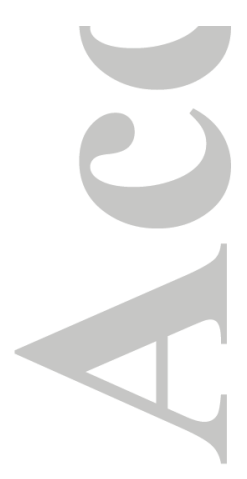

$170 \times 130 \mathrm{~mm}(300 \times 300 \mathrm{DPI})$ 